\title{
Modelo para la Adopción de Cloud Computing en las Pequeñas y Medianas Empresas del Sector Servicios en Medellín, Colombia
}

\author{
Juan C. Patiño-Vanegas ${ }^{(1) \star}$ y Alejandro Valencia-Arias ${ }^{(2)}$ \\ (1) Facultad de Ciencias Económicas y Administrativas, Instituto Tecnológico Metropolitano - ITM, calle 73 № 76 A - \\ 354 Vía El Volador, Medellín - Colombia. (e-mail: juanpatino@itm.edu.co) \\ (2) Facultad de Ingeniería, Corporación Universitaria Americana - CUA, Cra. 42 No. 52 - 06, Medellín - Colombia. \\ (e-mail: jvalencia@americana.edu.co)
}

* Autor a quien debe ser dirigida la correspondência.

Recibido Ene. 25, 2019; Aceptado Mar. 29, 2019; Versión final Jun. 12, 2019, Publicado Dic. 2019

\begin{abstract}
Resumen
Se presenta y analizan para examinar los factores de aceptación de Computación en la Nube (Cloud Computing) en las pequeñas y medianas empresas del sector servicios en Medellín (Colombia), mediante el Modelo de Aceptación Tecnológica. La metodología fue exploratoria descriptiva, ejecutando la investigación en dos fases, una de revisión de literatura, seguida por la aplicación de entrevistas a cinco individuos pertenecientes a organizaciones del sector servicios. Como resultado, surge un modelo de adopción de esta tecnología, delimitado por tres tipos de variables: estratégicas, de competitividad y desempeño; develando la utilidad y facilidad de uso percibida por los individuos frente a la Computación en la Nube, y por lo tanto la intención de uso. Aunque la relación con la Computación en la Nube está sujeta al entorno y las experiencias de las pequeñas y medianas empresas, el modelo contribuye de manera transversal al desarrollo tecnológico y la innovación basada en conocimiento.
\end{abstract} planeado

\section{A Model for the Adoption of Cloud Computing in Small and Medium-Sized Companies in the Service Sector in Medellín, Colombia}

\begin{abstract}
The acceptance factors of cloud computing in small and medium-sized companies in the service sector in Medellín (Colombia), through the Technology Acceptance Model are presented and analyzed. The methodology was exploratory and descriptive, executing the research in two steps: a literature review, followed by the application of interviews to five members belonging to organizations of the service sector. As a result, of this emerges a model of adoption of this technology, delimited by three types of variables: strategic, competitiveness and performance, showing to be useful and user friendly. Although the relation with the Cloud Computing depends upon the environment and the experiences of the small and mediumsized companies, the model contributes in different ways to technology development and to knowledgebased innovation.
\end{abstract}




\section{INTRODUCCIÓN}

La computación en la nube es un anglicismo que surge de los términos Cloud (o nube, palabra que se usa generalmente para representar la Internet) y Computing (computación en español, que alude a los sistemas informáticos) (Armbrust et al., 2009; Lasica, 2009). Se define como un modelo que permite acceso a la red bajo demanda a un conjunto de recursos informáticos (redes, servidores, almacenamiento, aplicaciones y servicios) que pueden ser rápidamente aprovisionados y liberados con un esfuerzo de gestión mínimo (Mell y Grance, 2011)y hoy se presenta como un nuevo paradigma para representar las capacidades informáticas como un servicio para compartir recursos en internet (Farrag et al., 2019). En el sentido más simple, se refiere a los servicios y aplicaciones que operan a través de Internet con proveedores que cuentan con todas las medidas de seguridad necesarias, tanto físicas como de software, de forma que estén disponibles para el usuario, permitiendo encontrar todo tipo de utilidades: servicios, aplicaciones, almacenamiento, mail, gestión de información, backup, entre otros (Shetty y Kumar, 2015; Hosseini et al., 2019)Que, si se usa de manera significativa, puede mejorar los enfoques tradicionales en las empresas (Gao y Sunyaev, 2019). Al respecto, cabe destacar que el internet es un eje central en el desarrollo de las empresas, donde la computación en la nube se convierte progresivamente en una herramienta que transforma digitalmente a las organizaciones más exitosas (Cabarcas et al., 2012). Frente a esto, las organizaciones de todo el mundo están migrando rápidamente sus servicios e infraestructura de $\mathrm{TI}$ a los centros de datos en la nube para reducir los costos y garantizar una alta disponibilidad, escalabilidad y seguridad (Santos et al., 2019).

Sin embargo, la difusión de una tecnología no asegura su uso, aceptación y utilidad; es por esto que a través de los años se han desarrollado modelos para entender como una tecnología se propaga a través del tiempo que siendo importantes no logran hacerlo o no tienen éxito (Duque-Jaramillo y Villa-Enciso, 2016). Por ello, es importante identificar los factores que condicionan la adopción de la computación en la nube en las PYME - Pequeña y mediana empresa-, de tal manera que pueda identificarse su nivel de conocimiento, motivaciones y expectativas; planteando nuevas estrategias para su adopción. En este sentido, el presente estudio propone develar la forma como este tipo de organizaciones en el contexto colombiano incorporan estas tecnologías desde la aplicación del Modelo de Aceptación Tecnológica (TAM). Las investigaciones sobre innovación se enfocan desde dos puntos de vista: la difusión y la adopción. La difusión es el proceso mediante el cual una innovación es comunicada en el tiempo, a través decanales entre los individuos de un sistema social (Rogers, 1995). Mientras que la adopción está enfocada en determinar la tasa y la cantidad de adoptadores en una población durante un período de tiempo determinado (Montalvo y Kemp, 2008); debido a que un individuo puede tener conocimiento frente a la existencia de una innovación, pero no haber desarrollado una actitud favorable hacia la misma; es decir, haberla adoptado o rechazado. Frente a las teorías existentes para la difusión de innovaciones, emerge el Modelo de Aceptación Tecnológica (TAM) (Yong, 2004; Yong et al., 2010), basada en modelos anteriores, como son la Teoría de Acción Razonada (TRA) y la Teoría del Comportamiento Planeado (TPB) (Moreno-Agudelo y Valencia-Arias, 2017). En cuanto a la relación de la primera teoría (TRA), ésta sugiere que el comportamiento de un individuo está determinado por su intención previa a adoptar dicho comportamiento, y que esta intención está determinada, a su vez, por su actitud hacia el comportamiento en cuestión y por las normas subjetivas (Venkatesh et al, 2003).

También está la actitud como referencia a la evaluación positiva o negativa que realiza el individuo sobre el comportamiento, y está compuesta por las creencias sobre los resultados esperados para desempeñar el comportamiento; y las normas subjetivas. Adicionalmente, hacen referencia a lo que el sujeto cree que otros pares pensarían si él desempeñara el mismo comportamiento (Yong et al., 2010). Respecto a la segunda teoría (TPB), ésta plantea un esfuerzo por ampliar el rango de comportamientos explicados por el TRA teniendo en cuenta un constructo adicional: el control comportamental percibido. Este control da cuenta de qué tan fácil o difícil encuentra el individuo desempeñar el comportamiento, es decir, qué tan bien cree poder desempeñarlo (Benítez y Benavides, 2012). En este sentido, el TAM adiciona al modelo los constructos de utilidad y facilidad percibida, como antecedente directo de la intención de usarla (Davis, 1989; Yong et al., 2010). Respecto al primer constructo (utilidad percibida), se refiere al grado en que una persona cree que usando un sistema en particular mejorará su desempeño. Para el segundo constructo (facilidad percibida) hace referencia de, hasta qué grado una persona cree que usando un sistema en particular realizará menos esfuerzo para realizar sus actividades (Yong, 2004). Así mismo, el Modelo de Aceptación Tecnológica adapta el TRA enfocado en la conducta de uso de nuevas tecnologías, por lo que, desde esta perspectiva, el TAM es un modelo que explica claramente el comportamiento de adopción de las tecnologías (Villa et al., 2018). Aunque el TAM ayuda a conocer si una tecnología va ser utilizada de manera óptima, es fundamental identificar las variables externas que influyen directamente en los constructos principales (utilidad y facilidad de uso), para determinar la relación hacia el uso de dicha tecnología. 


\section{METODOLOGÍA}

Se realizó un estudio exploratorio descriptivo, con el objetivo de examinar los factores de aceptación de la computación en la nube en las PYME del sector servicios en la ciudad de Medellín (Colombia). a través del Modelo de Aceptación Tecnológica (TAM). Se tomó una muestra en 5 empresas con el fin de realizar un análisis a profundidad que permitiera conocer el conjunto de creencias y normas que orientan el comportamiento organizacional desde sus principios y valores. Además de tener una visión holística para comprender el proceso de adopción; que a su vez servirá de insumo para proponer estrategias empresariales internas que puedan ser empleadas en las pequeñas y medianas empresas. Por tanto, la investigación se desarrolló en dos fases. En la primera, se obtuvo información secundaria a través de fuentes información científica como, Scopus, Science Direct, Gardner, IEEE y E- Journal; lo cual permitió agrupar variables en categorías de análisis y develar el estado del arte y modelos más importantes en la temática abordada. En la segunda fase, se construyó un instrumento de entrevista semiestructurada para obtener información de fuentes primarias. Aplicadas a 5 PYME de la ciudad de Medellín, cuya información permitió identificar las causas relacionadas los elementos que influencian en la adopción de la computación en la nube en este tipo de organizaciones, y definir las variables externas del Modelo de Adopción Tecnológica (TAM); así mismo, la aplicación de las entrevistas generó los insumos para la proposición de estrategias favorables para la aceptación de esta nueva tecnología en las pequeñas y medianas empresas.

Igualmente, para la realización de las entrevistas, los participantes fueron informados con respecto al objetivo del estudio, su alcance, riesgos, beneficios y demás aspectos clave que demandan los ejercicios investigativos donde se interactúa con seres humanos. Ello quedó manifiesto con la firma del consentimiento informado, donde se expresaron todos los aspectos mencionados, además el carácter meramente académico con el que se emplearía la información recopilada, así como la confidencialidad en datos que puedan identificar tanto al funcionario como a la organización que representa. La información recopilada durante esta fase fue grabada en audio con autorización de los informantes, cuyos registros fueron administrados por el equipo investigador y destruidos una vez que las narrativas obtenidas fueron transcritas y codificadas. Con dicha transcripción y codificación, se realizó el respectivo análisis, organizando los datos a través de mapas conceptuales, lo que permitió proponer el modelo de adopción de esta tecnología, delimitado por tres tipos de variables: estratégicas, de competitividad y desempeño como estrategia empresarial

\section{RESULTADOS Y DISCUSIÓN}

La figura 1 permite apreciar que las percepciones difieren entre la función de la organización y las personas que la dirigen. En este sentido, la visión de los entrevistados está vinculada al hecho que la implementación de las TIC no siempre es necesaria, pero reconocen la importancia de tenerla como eje central de la organización. Del mismo modo, se observa que las TIC van a la vanguardia con la innovación y tecnología, moviendo el mercado actual y permitiendo que la organización pueda ver más allá, y dar diferentes valores a sus clientes. Adicionalmente, plantean que la incorporación de nuevas herramientas TIC son algo deseable, o un medio para lograr el mejoramiento económico de la organización. También hay cierto consenso en promover el crecimiento, relacionado al capital y músculo financiero que promueva la inversión en infraestructura, equipos y nuevas tecnologías. Sin embargo, las motivaciones se centran en mayor medida a invertir en lo que realmente les interesa a la PYME, dejando de un lado las Tecnologías de la Información y la Comunicación. Caso contrario como lo indican varios autores, utilizar este servicio ha sido de gran utilidad para el sector empresarial debido a que plantea mejoras, evoluciones o nuevos entornos con inversiones bajas, lo que permite a las organizaciones ganar flexibilidad y agilidad.

Dentro de este panorama, las creencias determinan que la computación en la nube es el pilar fundamental en la gestión de la empresa para contribuir en el posicionamiento en el mercado. Por lo tanto, dos de las empresas participantes del estudio, indican que esta tecnología es un sistema que ofrece ventajas competitivas para gestionar la información y promover la movilidad entre los empleados. Sin embargo, consideran que es más estratégico tercerizar el servicio que utilizar recursos propios (infraestructura, personal, capacitación). Mientras que otra empresa sostienen que no usan la computación en la nube porque es una tecnología que está en etapa de crecimiento y que aún le falta tiempo en el mercado para ser mejorada; por lo que aún no percibe la necesidad de incorporarla en sus procesos internos. Por lo tanto, la computación en la nube es visto por los entrevistados como una tendencia para almacenar la información que va en crecimiento, que permite fortalecer la continuidad del negocio bajo las necesidades de cada empresa que proveen soluciones integrales. También, manifiestan que es la evolución de la infraestructura informática tradicional hacia servicios de computación centralizados en internet, estructuradas a las necesidades de cada empresa garantizando agilidad tecnológica. Coincidiendo que la adopción de esta tecnología, trae consigo ventajas organizacionales, como la administración, el acceso, la seguridad y la 
posibilidad de adquirir servicios con proveedores externos que ofrezcan la tecnología. Del mismo modo, autores como Archibald (2015), manifiestan que los servicios cloud existentes permiten a las empresas tener disponibilidad de integrar su infraestructura, software y diversos tipos de soluciones, logrando un flujo de caja predecible y evitando altas inversiones en equipos tecnológicos.

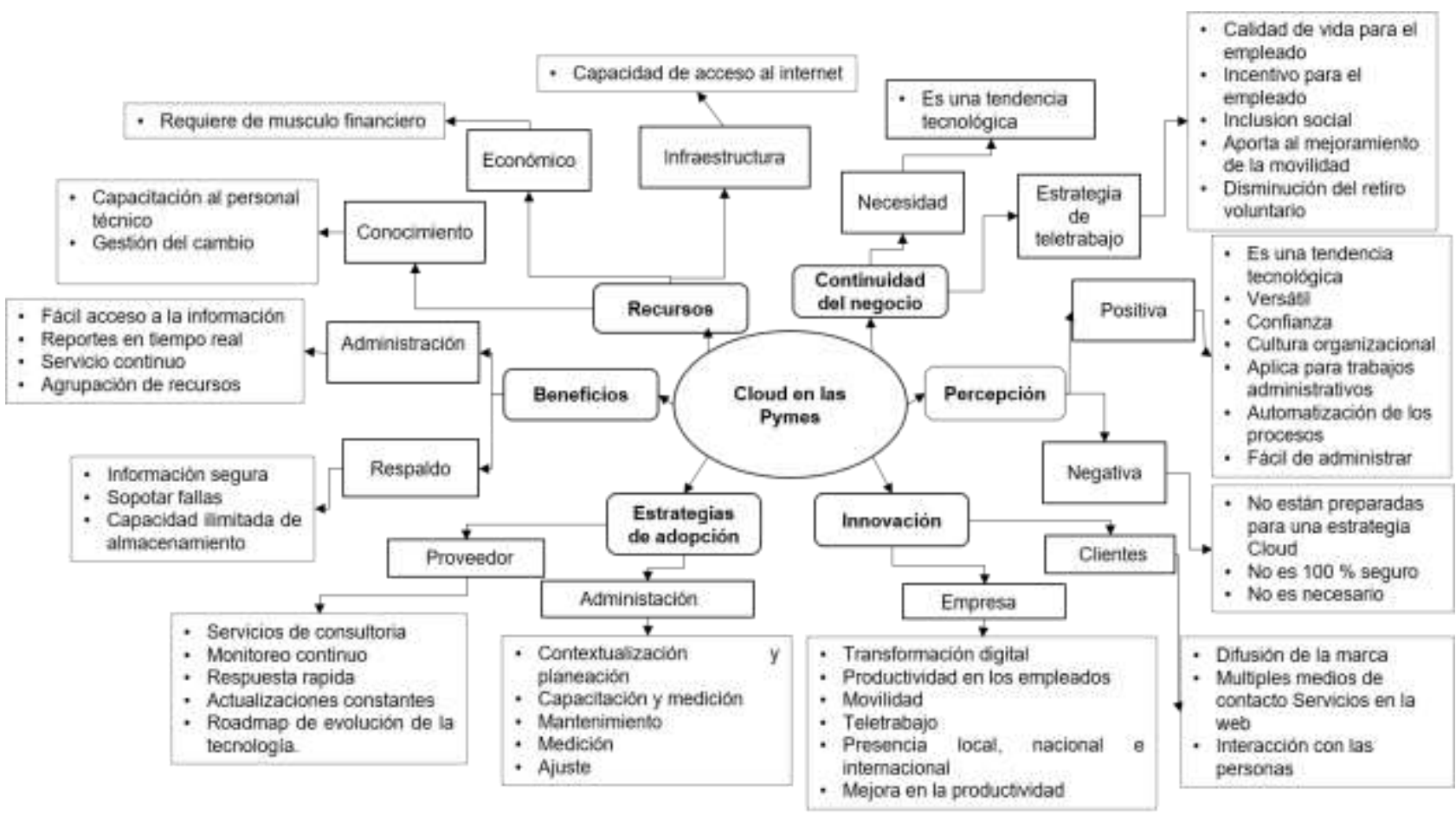

Fig. 1: Elementos estratégicos de la computación en la nube

En este mismo sentido, los entrevistados coinciden que es una tecnología que les ofrece beneficios para facilitar su trabajo y centrarse en lo que realmente es el negocio, ahorrando esfuerzos en la administración de recursos tecnológicos. Respecto a este planteamiento, autores como Kim et al., (2008), aseveran que toda acción electrónica genera confianza en el comportamiento de uso de una persona en la medida en que perciba seguridad. Dicha confianza contribuye en el comportamiento de adopción de la tecnología y favorece el desarrollo de actitudes hacia su uso, reduciendo la percepción de riesgo que presentan las actividades electrónicas para los usuarios en términos de divulgación de información personal, seguridad de los datos almacenados en el dispositivo o seguridad para realizar transacciones desde el mismo. En su mayoría, las PYME perciben la computación en la nube como algo que en corto plazo se debe adoptar, aunque también consideran alto el grado de incertidumbre en la utilización del servicio y prefieren su modelo convencional debido a que todavía en el mercado existe desconocimiento de esta herramienta. Sin embargo, pueden existir varias razones para esto, una de ellas que las empresas no estén interesadas en el tema o no haya surgido la necesidad de utilizarla, otra podría ser que las compañías prestadoras del servicio no se den a conocer de la manera adecuada. Al respecto, en la figura 1 puede apreciarse también, como la mayoría de las pequeñas y medianas empresas son conscientes de la importancia que esta tecnología ofrece para incrementar la innovación y acelerar su ciclo de experimentación en la administración de los recursos informáticos a bajo costo, mejorar la eficiencia, flexibilidad y proteger la información.

Entre los factores importantes se relaciona la facilidad de adaptación tecnológica, la administración de la información, y los escenarios en los cuales el uso de servicios de Cloud Computing impacta positivamente en el negocio y ayude a establecer la relación de costo/beneficio al momento de migrar desde una plataforma tradicional hacia la nube. Las expectativas están enfocadas a que cada empresa desea estar a la vanguardia para fortalecer la empresa y ganar flexibilidad y agilidad. Tal como lo refirió uno de los participantes del estudio: "Estar a la vanguardia y ser un pionero innovador en tecnología, ser una un empresario proactivo y a la vez precavido a la hora del manejo de la información propia y como tal y obviamente creo que ayudaría mucho a replicar esto a nivel de la organización a las demás personas para que aporten o todos vayan bajo un mismo norte que es sacar esto adelante y obviamente todo el tema de la tecnología e innovación siempre va hacer un puente para mejorar el futuro de la empresa". Adicional a lo ya expuesto, de la figura 1 puede inferirse también que migrar servicios empresariales a entornos Cloud Computing no es una tarea fácil, cada empresa es un universo diferente y tiene necesidades diferentes. Por lo tanto, la administración de la información es un factor determinante para promover la innovación, ya que, si se gestiona desde cualquier momento y lugar, se erige como una herramienta para implementar al interior de las organizaciones. 


\section{Propuesta de modelo estratégico}

Entender mejor la dinámica de aceptación de la computación en la nube, y su valor en el contexto de economías emergentes ha dado como resultado el planteamiento de un modelo estratégico -tal como se puede apreciar en la figura 2, para favorecer la aceptación de esta tecnología, a través del conocimiento técnico identificado en las PYME a la hora de asumir decisiones; lo cual a su vez ha permitido entender cuáles son los factores externos motivacionales que influencian este tipo de organizaciones a adoptar la tecnología y promover la difusión de la modernización y la globalización de estas. Así, eestá herramienta, se constituye por tanto en una identificación de la apropiación crítica de los mecanismos de desarrollo, buscando expresar que la modernización no debe estar enfocada en adquirir equipos nuevos sin antes reconocer los beneficios que estos ofrecen a las empresas, particularmente las pequeñas y medianas.

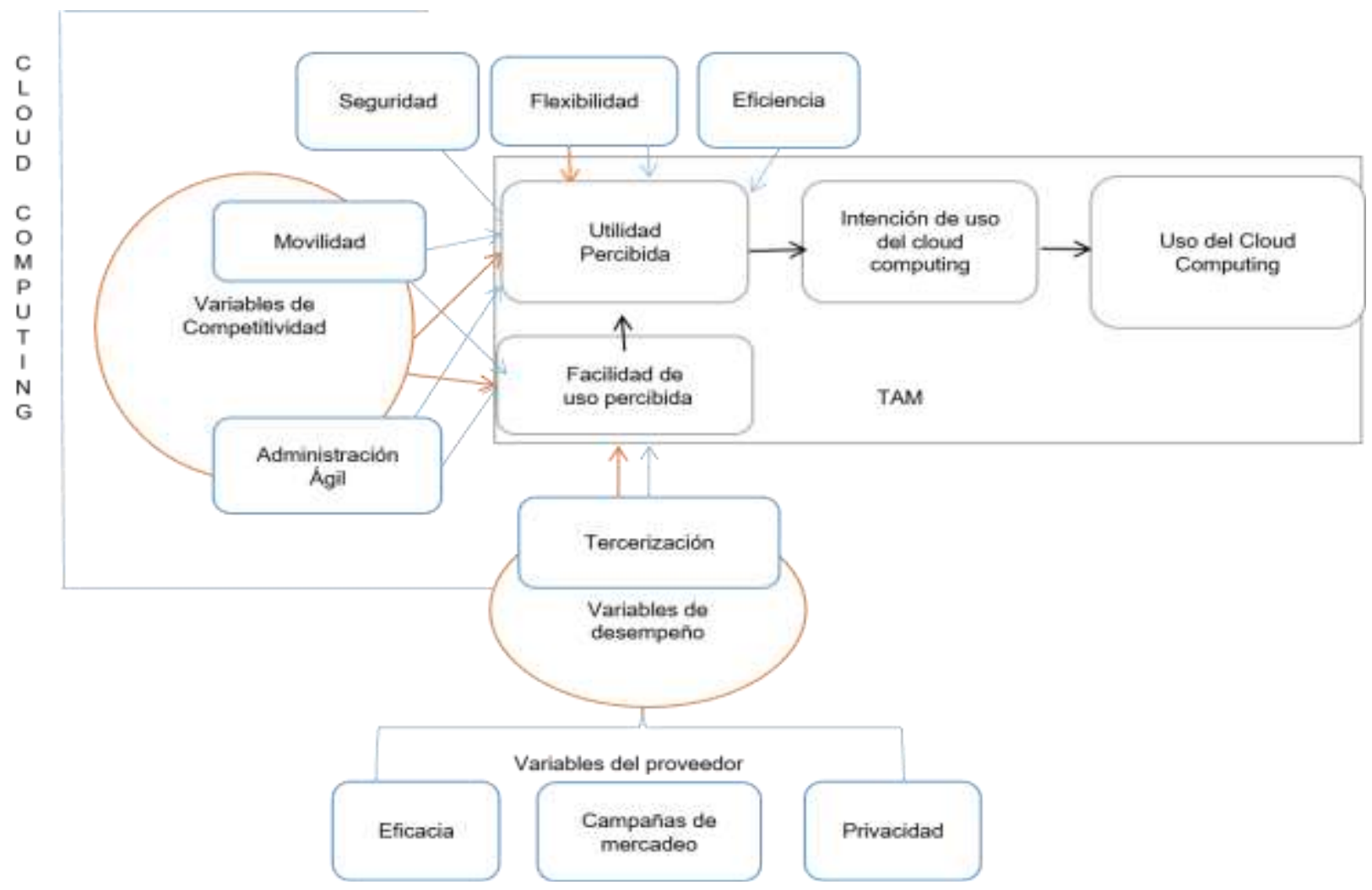

Fig. 2: Propuesta de modelo estratégico de adopción de la computación en la nube en las PYME.

Con base en lo anteriormente, se definieron tres variables macro: estratégicas, competitividad y desempeño, que están orientadas a gestionar lo mejor posible los recursos tecnológicos y la toma de decisiones. Adicionalmente, se delimitaron factores secundarios que fortalecen las variables macro, como aquellos que son fundamentales para que las PYME decidan adoptar la tecnología. En cuanto a la variable estratégica se definen tres factores secundarios: seguridad, eficiencia y flexibilidad; que se fundamentan en que la computación en la nube debe ofrecer al usuario mecanismos que aseguren la integridad de la información y contribuyan a la flexibilidad de la empresa, y a su vez, debe permitir una alineación a los procesos internos con el mínimo de recursos posibles. Por lo tanto, influyen sobre el constructo principal del modelo base: la utilidad percibida, debido a que hay una influencia directa hacia la Intención de uso y una indirecta en el uso de la tecnología. Esto significa, que, al percibir la computación en la nube como un elemento estratégico dentro de la organización, impulsa a una actitud de uso que con lleva a la aceptación de la misma.

Este modelo permite tener una mejor comprensión de las variables externas que se deben tomar en cuenta al momento de analizar el proceso de implementación y apropiación de la computación en la nube en las actividades habituales de la empresa. Por ende, contar con una estrategia apropiada de Cloud Computing permite a las empresas gestionar manera correcta los procesos internos. Sin embargo, el solo hecho de implementar una nueva tecnología no asegura su uso, desempeño y utilidad. Lo que significa, que uno de los mayores retos en la administración tecnológica, radica por tanto en la selección de la tecnología apropiada en virtud de la abundancia de nuevas TIC emergentes con el desarrollo global y sistemas que posibiliten entregar soluciones informáticas a la rapidez que requieren los actuales negocios. Sin embargo, Fernández, McAnally y Vallejo, (2015) manifiesta que no es precisamente la adquisición de tecnología la que está relacionada con una conciencia tecnológica, sino el conjunto de prácticas que se desarrollan alrededor de ellas. 


\section{Proceso estratégico para favorecer la computación en la nube}

Para inducir dinámicas de innovación se requiere planificación y acción articulada con los procesos internos de una empresa, por lo que es necesario contar con una estrategia que surja de trabajar con y contra lo incierto, lo aleatorio y la incertidumbre. Por tanto, la implementación exitosa de la estrategia necesita una visión global combinada con un enfoque pragmático. Frente a esto, en la figura 3 se determina los pasos correctos que una empresa debe seguir hacia la adopción y el éxito de la computación en la nube.

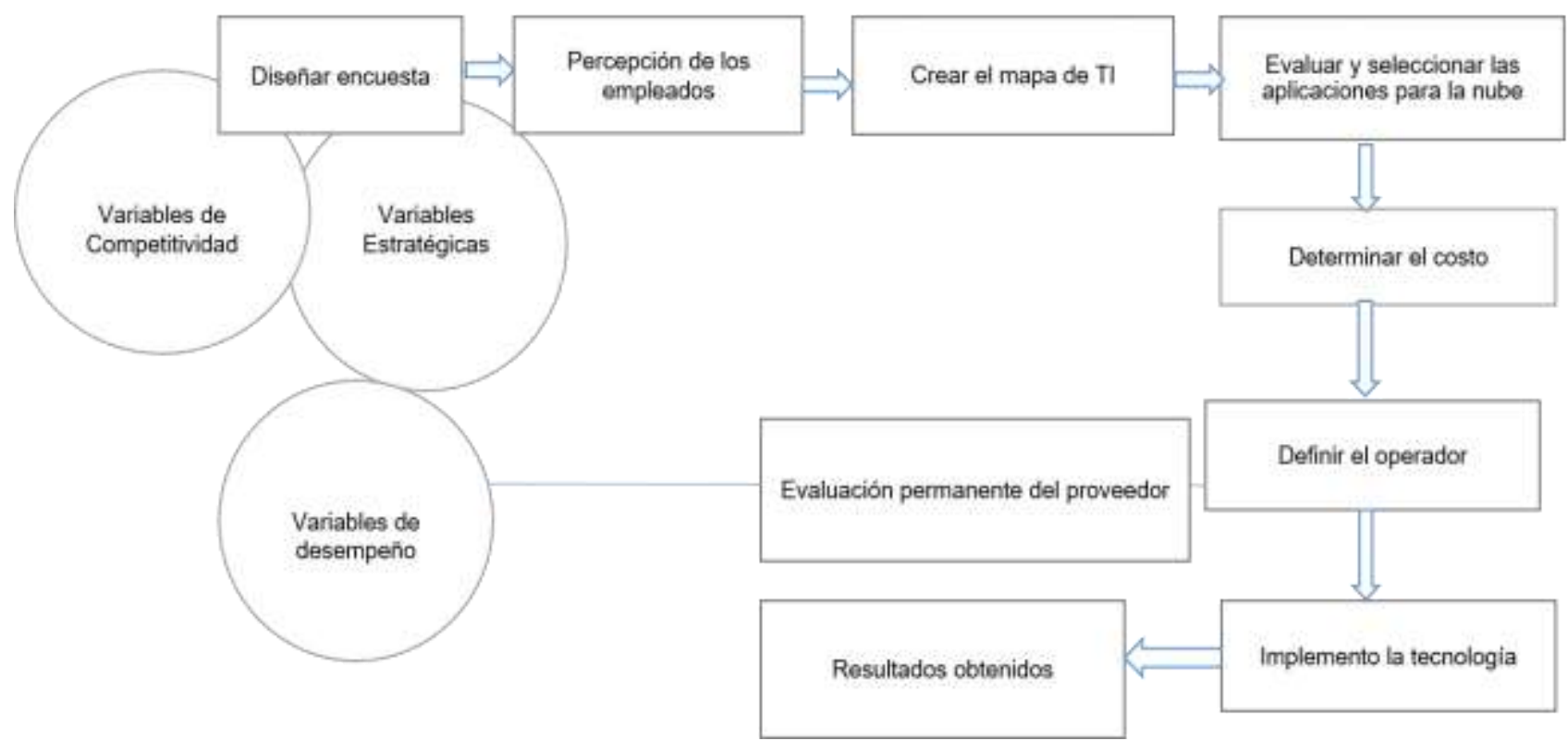

Fig. 3: Proceso estratégico para el favorecimiento de la computación en la nube.

\section{Crear la estrategia y el mapa de ti}

Diseñar un mapa de la estructura TI permita reconocer el estado actual de la misma como: el tipo de hardware, software, almacenamiento de la información, canal de internet, cantidad de oficinas remotas y plan de continuidad ante pérdidas de información. De esta manera, se pueden reconocer los elementos con los que cuenta la empresa y con los que podrá tener continuidad en el mercado en los próximos años. En este sentido, los empleados usan diferentes medios para comunicarse entre ellos, $y$ en algunas ocasiones almacenan información personal y de la empresa en la nube por cuenta propia sin importar si cuentan con el apoyo del departamento de Tl; sin embargo, esta actividad tiene algunas desventajas entre las que se incluye la falta de administración central, control de sus accesos y fuga en la información. Por lo tanto, es necesario validar a qué tipo de información se le permite acceder a los empleados desde sus dispositivos personales y buscar la manera de unirlas y llevarlas a un entorno virtual sobre internet controlado por la empresa.

Frente lo anterior, se debe proceder a evaluar y seleccionar las aplicaciones para la nube. Seguidamente, se tendrá que realizar un análisis o diagnóstico global de la empresa (Estrategias, objetivos y estructura) y un diagnostico funcional ( $+\mathrm{D}$, producción, finanzas, empleados y aspectos generales de los recursos humanos) de tal forma que se identifique la proyección y las metas a las que quiere llegar la organización. Luego, extraer ventaja de un ambiente tecnológico ágil para identificar qué es lo que quiere hacer (aprovechar una oportunidad o neutralizar una amenaza) de tal forma que se reconozcan las características de las arquitecturas orientadas a los servicios como laaS -Loggin as a service en su acepción en inglés-, PaaS -Platform as a service- y SaaS -Software as a service-; que ofrecen un ambiente tecnológico ajustable a cualquier tamaño de empresa y que contienen todos los mecanismos necesarios para integrar la información y cambiar de una manera eficiente con los mínimos recursos posibles. Finalmente, el último aspecto a considerar, es la identificación de las aplicaciones con las que cuenta la empresa, de tal forma que puedan ser priorizadas y estandarizarlas en una sola. Al respecto, resulta más factible migrar las aplicaciones que sean susceptibles a la estandarización, aquellas que sean independientes o que tengan una arquitectura orientada a servicio a través de la nube. 


\section{Determinar la estructura de la computación en la nube}

Una vez que se hayan identificado las aplicaciones con las que cuenta la empresa, se podrá establecer la forma adecuada para llevarlas a nube pública, privada o una híbrida formada por ambas. En cuanto a las nubes públicas, la infraestructura y el software están ubicadas físicamente en las sedes del proveedor, presentando menos riesgos y un alto retorno de inversión (ROI).Adicionalmente, incluye almacenamiento de información, escritorios virtuales, infraestructura, audios y videos en la web, entre otros. Para las nubes privadas, los datos y la infraestructura se entregan en la misma empresa y es necesario mayor conocimiento de la misma. Adicionalmente, cabe destacar que este tipo de nubes ofrecen un ROI de mayor potencial y menor riesgo tal como lo expone Cabarcas y Marrugo (2012) en su estudio. Para las empresas que se dedican a desarrollar su propio software, son considerados actividades de trabajo con buenas probabilidades tanto para la nube pública como para la privada, y para las pequeñas y medianas empresas, son más convenientes las nubes publicas porque se perciben mejores beneficios económicos y luego de identificar el tipo de nube que se quiere implementar es necesario definir el tipo de arquitectura a usar (laas, Paas, Saas).

\section{Determinar el costo}

Reconocer el retorno de la inversión (ROI) que la nube puede ofrecer, teniendo en cuenta el tiempo para la implementación inicial, es una actividad importante. Al determinar el ROI, es necesario iniciar calculando el ahorro que se alcanzará en todas las dimensiones del área de TI relacionadas con las aplicaciones, por ejemplo, el costo del hardware, licencias y las renovaciones del software, la gestión del usuario final y el soporte a las aplicaciones. Adicionalmente se puede calcular el ROI asociadas al negocio, tales como el aumento en la productividad de los empleados y el uso de los recursos; con el fin de evitar gasto del capital, y la disminución del riesgo debido a una mejora en la disponibilidad (Salcedo y Vargas, 2010).

Por otro lado, se puede validar el panorama completo con respecto al TCO (costo total de propiedad) para estimar el costo de sus aplicaciones menos efectivas actuales mediante el cálculo del impacto en los ingresos o de los porcentajes de satisfacción y su impacto en el negocio Lo que significa, que se debe tomar en cuenta el resultado de la encuesta del modelo para darse una idea de lo que piensan los empleados de lo que será la computación en la nube en su organización.

\section{Selección del proveedor}

Los proveedores de servicios de internet están produciendo capacidades de anchos de banda más amplios a precios más bajos, lo cual coloca los servicios de la computación en la nube al alcance del mercado. También el portafolio es amplio con una gama de soluciones que ofrecen alternativas de crecimiento. Por lo tanto, es conveniente contar con un proveedor que tenga presencia local por temas de agilidad ante cualquier falla, acompañamiento en consultoría y que lleve a la empresa a una dirección que proteja de la necesidad de realizar cambios costosos e inesperados de tecnología en el futuro (Alonso, 2007).A partir de esto, se sugiere diseñar un proyecto basado en la visión de la empresa y las características antes mencionadas que permita obtener beneficios de expansión del negocio, la movilidad de empleados y el teletrabajo. Estos movimientos estratégicos pueden dar como resultado un departamento de $\mathrm{Tl}$ cuyas aplicaciones y ambientes sean de mayor confianza y seguridad, lo cual se traduce en que el resto de la organización crea más en las capacidades de la tecnología en pro de cumplir los objetivos organizacionales.

\section{DISCUSIÓN FINAL}

Las PYME que participaron, no cuentan con actividades de planificación para recuperar y restaurar la pérdida de información ante un desastre físico o informático. Del mismo modo, no podrían ejercer la práctica del teletrabajo por las falencias que tienen en la gestión del cambio. Sin embargo, reconocen la importancia de estas actividades. Aunque estos cambios deben estar apoyados por un músculo financiero que promueva la utilización de estas prácticas, porque su economía es tan cambiante que no les permite mejorar sus recursos debido a que los proyectos son de corta duración, lo que conlleva a que adoptar nuevas tecnologías no sea una prioridad y centren sus esfuerzos a generar utilidades que es lo que realmente importa en su negocio.

De todo esto se desprende que, las pequeñas y medianas empresas reconozcan la versatilidad de la computación en la nube debido a la flexibilidad operativa y administrativa para gestionar la información de una manera eficiente. Igualmente, esta tecnología permite sustituir procesos manuales que consumen tiempo, como la compra de servidores o tener centro de datos internos en la empresa. Adicionalmente, es visto como una tendencia para almacenar la información que va en crecimiento, lo que permite fortalecer la continuidad del negocio bajo las necesidades de cada organización. Por otro lado, vale resaltar que el uso 
de métodos cualitativos permite comprender una mejor manera cómo las variables TAM interactúan entre sí para predecir el uso de la tecnología. También posibilitan identificar otras variables externas y plantear nuevos modelos. En este caso, se planteó el modelo estratégico para favorecer la aceptación de la computación en la nube como un elemento aplicable a diferentes sectores de la industria, debido a que sus variables externas están enfocadas en las ventajas que ofrece la tecnología como factor competitivo, la tranquilidad hacia el consumidor como factor estratégico y la gestión del proveedor como elementos de desempeño.

Así, con base al estudio desarrollado se puede concluir que el modelo estratégico propuesto es una herramienta que busca una adecuada integración del proceso productivo en las actividades de innovación tecnológica y a su vez está fundamentado desde las motivaciones y expectativas que influyen directamente al uso de la tecnología, que permite conocer la probabilidad de que ocurra la adopción del cloud computing. Por lo tanto, se identifican tres variables externas al modelo central definidas como estratégicas, competitivas y desempeño, que están asociadas a influenciar en los constructos principales; que a su vez tiene como factores centrales la eficiencia, seguridad, flexibilidad, movilidad, administración ágil, tercerización como aquellos elementos externos de la aceptación del cloud computing.

Aunque el modelo se ha destacado a nivel mundial entre los modelos existentes por su aplicabilidad en diferentes contextos, posibilita identificar que determina la adopción de las TIC. Como es el caso del uso del internet móvil en la universidad de Salamanca España (Sanchez et al., 2017) adopción de nuevas tecnologías en instituciones de educación superior en Chile (González y Peralta, 2015), adopción de software libre en las pymes de Medellín-Colombia) entre otras. Cabe resaltar que, se vuelve riesgoso plantear modelos generales con factores que no responden de forma apropiada a las necesidades del entorno (Valencia-Arias et al., 2018), es decir, que los fenómenos existentes en una región o un contexto especifico se manifiestan de manera irregular comparados con otras zonas geográficas y entenderlos son un insumo importante para plantear nuevas formas de gestión en las empresas. Aun así, no se garantiza que puedan ser aplicados en otros contextos. Frente a esto, el modelo que se propone en esta investigación fue aplicado en Pymes de la ciudad de Medellín del sector servicios respondiendo a las necesidades de su contexto y entorno cultural. Por otro lado, determinar los pasos correctos hacia la adopción es fundamental si se requiere asegurar el uso de la tecnología. Frente a esto, es esencial diseñar una estrategia de capacitación que incluya temas asociados al crecimiento y posicionamiento de la empresa (Clientes y proveedores), la facilidad de uso para gestionar la información y la utilidad para mejorar los tiempos en los procesos. De esta manera el mecanismo de capacitación favorece la confianza entre el empleado y la tecnología; el desarrollo de las habilidades para el uso, la disminución de los temores ante el cambio y la confianza del trabajador para promover la productividad empresarial.

\section{CONCLUSIONES}

De acuerdo al trabajo presentado y a los resultados obtenidos, se pueden plantear las siguientes conclusiones principales:

El uso del modelo puede generar nuevas formas de dirección que involucren la integración de la tecnología en los objetivos estratégicos de la PYME aumentando su productividad desde el entendimiento de las necesidades internas y externas para tomar la decisión correcta sobre el tipo de cloud computing adoptar. Teniendo en cuenta su versatilidad, flexibilidad operativa y administrativa para que impacten estratégicamente en procesos más eficientes.

Por tanto, el modelo propuesto plantea variables externas al modelo inicial del TAM como mecanismo para disminuir los niveles de incertidumbre que permita a las PYME una mejor planificación en el proceso de adopción tecnológica fundamentado en tres variables; estratégicas, de competitividad y desempeño como componentes que permitan resaltar las bondades de la tecnología, la flexibilidad en la administración y la articulación con los proveedores externos que garanticen procesos más eficientes. A su vez

También las PYME que participaron en el estudio reconocen que la dinámica del mercado es cambiante, en especial las exigencias de sus clientes y los negocios que los obliga a incorporar las TIC en sus procesos internos para responder a las necesidades externas del mercado. Reconociendo también que su relación al uso está sujeta al entorno y a las experiencias previas que se haya tenido con la tecnología. Lo que permite conocer la probabilidad de que ocurra la adopción.

\section{REFERENCIAS}

Armbrust, A., R. Fox, R. Griffith. y J. D. Randy, Above the Clouds: A Berkeley View of Cloud Computing, Berkeley Tech. Rep. UCB , 07-013 (2009) 
Benítez, LI. N. y C. G. Benavides, Investigación en Innovación Tecnológica: un Estudio Bibliométrico, Revista Europea de Dirección y Economía de la Empresa, 21(2), 157-168 (2012)

Cabarcas, A., R.J. Martelo. y P. Puello, TICS en las PYMES: Herramienta Útil para Aumentar la Competitividad, Ingeniator, 3(5), 10-17(2012)

Cabarcas, A., P.P. Marrugo. yR. Canabal, Cloud Computing: Tecnología Verde Como Estrategia Para La Responsabilidad Social Empresarial, Saber Ciencia y Libertad, 7(2), 1794-7154 (2012)

Sanchez, J.C., S. Olmos y F. García-Peñalvo, ¿Utilizarán los futuros docentes las tecnologías móviles? Validación de una propuesta de modelo TAM extendido, RED. Revista de Educación a Distancia,52(5), 30 (2017)

Davis, F.D, Perceived Usefulness, Perceived Ease of Use, and User Acceptance of Information Technology,MIS Quarterly: Management Information Systems, 13(3), 319-339 (1989)

Duque-Jaramillo, J. C., y E.M. Villa-Enciso, Big Data: desarrollo, avance y aplicación en las Organizaciones de la era de la Información, Revista CEA, 2(4), 27-45 (2016)

Farrag, A. A., S.A. Mohamad y E. El-Horbaty, Swarm Optimization for Solving Load Balancing in Cloud Computing, doi: 10.1007/978-3-030-14118-9_11, Springer Verlag, 921,102-113 (2019)

Fernandez, K., L. McAnally. y A. Vallejo, Apropiación Tecnológica:Una Visión Desde los Modelos y las Teorías que la Explican, doi: 10.4151/07189729, Perspectiva Educacional, 54(2), 109-125 (2015)

Gao, F. y A. Sunyaev, Context matters: A review of the determinant factors in the decision to adopt cloud computing in healthcare, doi: 10.1016/j.ijinfomgt.2019.02.002, International Journal of Information Management, 48, 120-138 (2019)

González, B. y P. Valdivia, Posibilidades para el uso del modelo de aceptación de la tecnología (TAM) y de la teoría de los marcos tecnológicos para evaluar la aceptación de nuevas, Revista Electrónica Educare, 19(2) 181-196 (2015)

Hosseini, S., G. Fallon., V. Weerakkody y U. Sivarajah, Cloud computing utilization and mitigation of informational and marketing barriers of the SMEs from the emerging markets: Evidence from Iran and Turkey, doi:10.1016/j.ijinfomgt.2018.11.011, International Journal of Information Management, 46, 54-69 (2019)

Kim, D.J., D.L. Ferrin y H. R. Rao, A Trust-Based Consumer Decision-Making Model in Electronic Commerce: The Role of Trust, Perceived Risk, and their Antecedents, Decision Support System, 44(2), 544-564 (2008)

Mell, P. y T. Grance, The NIST Definition of Cloud Computing Recommendations of the National Institute of Standards and Technology, National Institute of Standards and Technology, 800-145 (2011)

Moreno-Agudelo, J. A. yJ.A. Valencia-Arias, Factores implicados en la adopción de software libre en las Pyme de Medellín,Revista CEA, 3(6), 55-75 (2017)

Rogers, E. M., The Diffusion of Innovation, $3^{\text {rd }}$ Ed. Newyork Free Press, New York (1995)

Santos, G. L., D. Rosendo y otros cinco autores, A Methodology for Automating the Cloud Data Center Availability Assessment, doi:10.1007/978-3-030-15032-7_85, Springer Verlag, 926, 1011-1023 (2019)

Shetty, J. y K. Kumar, Cloud Computing: An Exploratory Study on Adoption among SME Clusters in Bangalore and Mysore, Indian Journal of Science and Technology, 8(54), 169-175 (2015)

Villa, E.M., L. Ruiz., J.A. Valencia. y E. Picón, Electronic commerce: Factors involved in its adoption from a bibliometric analysis, Journal of Theoretical and Applied Electronic Commerce Research, 13(1), 39-7 (2018)

Valencia-Arias, J.A., S. Chalela-Naffah y J. Bermudez, A proposed model of e-learning tools acceptance among university students in developing countries, doi: 10.1007/s10639-018-9815-2, Education and Information Technologies, 24(2), 1057-1071 (2018)

Yong, L.A., L.A. Rivas. y J. Chaparro, Modelo de Aceptación Tecnológica (TAM): un Estudio de la Influencia de la Cultura Nacional y del Perfil del Usuario en el uso de las TIC,INNOVAR. Rev, 20(36),197-204 (2010)

Venkatesh, V., M.G. Morris., G.B. Davis. y F.D. Davis, User Acceptance of Information Technology: Toward a Unified view, MIS Q. Manag. Inf. Syst., 27(3), 425-478 (2003) 
